\title{
Artificial Intelligence and Machine Learning in Prostate Cancer Patient Management-Current Trends and Future Perspectives
}

\author{
Octavian Sabin Tătaru ${ }^{1} \mathbb{1}$, Mihai Dorin Vartolomei ${ }^{2,3, *}$, Jens J. Rassweiler ${ }^{4}$, Oșan Virgil ${ }^{1}$, Giuseppe Lucarelli ${ }^{5}{ }^{\circledR}$, \\ Francesco Porpiglia ${ }^{6}$, Daniele Amparore ${ }^{6}$, Matteo Manfredi ${ }^{6}$, Giuseppe Carrieri ${ }^{7}$, Ugo Falagario ${ }^{7}$, \\ Daniela Terracciano ${ }^{8}\left(\mathbb{D}\right.$, Ottavio de Cobelli ${ }^{9,10}$, Gian Maria Busetto ${ }^{11} \mathbb{(}$, Francesco Del Giudice ${ }^{12} \mathbb{(}$ \\ and Matteo Ferro ${ }^{9}$ (i)
}

Citation: Tătaru, O.S.; Vartolomei, M.D.; Rassweiler, J.J.; Virgil, O.; Lucarelli, G.; Porpiglia, F.; Amparore, D.; Manfredi, M.; Carrieri, G. Falagario, U.; et al. Artificial Intelligence and Machine Learning in Prostate Cancer Patient Management-Current Trends and Future Perspectives. Diagnostics 2021, 11, 354. https://doi.org/10.3390/ diagnostics11020354

Academic Editor: Sameer Antani

Received: 16 January 2021

Accepted: 17 February 2021

Published: 20 February 2021

Publisher's Note: MDPI stays neutral with regard to jurisdictional claims in published maps and institutional affiliations.

Copyright: (c) 2021 by the authors. Licensee MDPI, Basel, Switzerland. This article is an open access article distributed under the terms and conditions of the Creative Commons Attribution (CC BY) license (https:// creativecommons.org/licenses/by/ $4.0 /)$.
1 The Institution Organizing University Doctoral Studies (I.O.S.U.D.), George Emil Palade University of Medicine, Pharmacy, Sciences and Technology from Târgu Mureș, 540142 Târgu Mureș, Romania; sabin.tataru@gmail.com (O.S.T.); osan.virgil@gmail.com (O.V.)

2 Department of Cell and Molecular Biology, George Emil Palade University of Medicine, Pharmacy, Sciences and Technology from Târgu Mureș, 540142 Târgu Mureș, Romania

3 Department of Urology, Medical University of Vienna, 1090 Vienna, Austria

4 Department of Urology, SLK Kliniken Heilbronn, University of Heidelberg, 74074 Heilbronn, Germany; jens.rassweiler@slk-kliniken.de

5 Department of Emergency and Organ Transplantation-Urology, Andrology and Kidney Transplantation Unit, University of Bari, 70124 Bari, Italy; giuseppe.lucarelli@inwind.it

6 Department of Urology, San Luigi Gonzaga Hospital, University of Turin, Orbassano, 10143 Turin, Italy; porpiglia@libero.it (F.P.); daniele.amparore@unito.it (D.A.); matteo.manfredi85@gmail.com (M.M.)

7 Department of Urology and Organ Transplantation, University of Foggia, 71122 Foggia, Italy; giuseppe.caririeri@unifg.it (G.C.); ugofalagario@gmail.com (U.F.)

8 Department of Translational Medical Sciences, University of Naples Federico II, 80131 Naples, Italy; daniela.terracciano@unina.it

9 Division of Urology, European Institute of Oncology (IEO)-IRCCS, 20141 Milan, Italy; ottavio.decobelli@ieo.it (O.d.C.); matteo.ferro@ieo.it (M.F.)

10 Department of Oncology and Haematology-Oncology, Università degli Studi di Milano, 20122 Milan, Italy

11 Department of Urology and Renal Transplantation, University of Foggia, Policlinico Riuniti of Foggia, 71122 Foggia, Italy; gianmaria.busetto@unifg.it

12 Department of Urology, Policlinico Umberto I, Sapienza University of Rome, 00185 Rome, Italy; francesco.delgiudice@uniroma1.it

* Correspondence: mdvartolomei@yahoo.com

Abstract: Artificial intelligence (AI) is the field of computer science that aims to build smart devices performing tasks that currently require human intelligence. Through machine learning (ML), the deep learning (DL) model is teaching computers to learn by example, something that human beings are doing naturally. AI is revolutionizing healthcare. Digital pathology is becoming highly assisted by AI to help researchers in analyzing larger data sets and providing faster and more accurate diagnoses of prostate cancer lesions. When applied to diagnostic imaging, AI has shown excellent accuracy in the detection of prostate lesions as well as in the prediction of patient outcomes in terms of survival and treatment response. The enormous quantity of data coming from the prostate tumor genome requires fast, reliable and accurate computing power provided by machine learning algorithms. Radiotherapy is an essential part of the treatment of prostate cancer and it is often difficult to predict its toxicity for the patients. Artificial intelligence could have a future potential role in predicting how a patient will react to the therapy side effects. These technologies could provide doctors with better insights on how to plan radiotherapy treatment. The extension of the capabilities of surgical robots for more autonomous tasks will allow them to use information from the surgical field, recognize issues and implement the proper actions without the need for human intervention.

Keywords: prostate cancer; biomarker; genomics; artificial intelligence; artificial neural network 


\section{Introduction}

Prostate cancer (PCa) is the third most commonly diagnosed cancer worldwide, after lung and breast cancer and the fifth cause of cancer-specific death in males [1]. Around 191,930 patients will be diagnosed with PCa in 2020 in the United States, with an estimated 33,330 deaths [2]. In the last few years, research was focused on diagnosis, prognosis and prediction of PCa outcomes taking a leap through the use of Statistics and Artificial Intelligence (AI). The use of computer-based learning models has become a predominant area of research in $\mathrm{PCa}$.

Artificial Neural Networks (ANN) have increasingly been used to build advanced prognostic models for PCa [3]. To train a machine learning model it is enough to acquire structured datasets including input variables and outcomes, with little knowledge of the $\mathrm{PCa}$ insights. For instance, several novel tools are available for screening and diagnosis of PCa such as genomics, magnetic resonance imaging (MRI) and biomarkers (exosomes and molecular imaging). In this scenario, AI may have a pivotal role, first in the interpretation of this enormous amount of data, second in the development of machine learning algorithms that may help urologists to reduce the number of unnecessary prostate biopsies without missing the diagnosis of aggressive PCa. Moreover, the use of genomics, AI and extracellular vesicles [4] (exosomes and cell-free DNA from body fluids), can provide a more reliable and rapid PCa test [5].

$\mathrm{AI}$ is defined as the ability of a computer to perceive the surrounding environment and make the same decisions as human intellect on an action, all this to reach a certain goal [6]. Machine learning (ML) is a subfield of AI and implies the creation and deployment of algorithms to analyze data and its properties and is not given a task specifically based on certain predefined inputs from the environment. ML techniques can mainly be classified according to the type of label and feature. For labeling, ML can be classified into three models such as supervised, unsupervised and reinforcement learning. For features, ML can be classified into handcrafted or non-handcrafted feature-based techniques [7]. Deep learning (DL) is a form of ML that enables machine devices (such as computers) to learn from experience and understand the environment in terms of a hierarchy of concepts. Computers gather experience in learning and a human does not need to pre-specify all the data to the computer [8]. Lately, deep convolutional neural networks (DCNNs), a modified type of AAN, have been proven to have high efficiency when applied to digitized images, a form of computer aided diagnosis (CAD) analysis. DCNNs allowed the automatic extraction of imaging features from digitized images in PCa [9] and it is now used to classify PCa and benign tissues with magnetic resonance imaging (MRI) [10]. In the last few years, DL has application in image classification, object detection, segmentation [11], the detection of anatomical and cellular structures, tissue segmentation, device aid on disease diagnosis and prognosis [12], with a new model emerging, which seems to perform better, called the massive-training artificial neural network [13]. Clinical decision support systems (CDSSs) are being developed to provide improvement in decision making. At this moment, reviews and systematic reviews give limited data on how ML and DL techniques could provide clinical application based on CDSSs in PCa oncological care [14,15]. Therefore, AI and ML are still being considered an area of development. A brief explanation and glossary of technical terms is provided in Table 1. 
Table 1. A glossary of Artificial Intelligence (AI) Terms.

\begin{tabular}{cc}
\hline Technical Term & Short Definition \\
\hline Artificial Intelligence (AI) & $\begin{array}{c}\text { Any technique that enables machines to mimic } \\
\text { human behavior [6] }\end{array}$ \\
\hline Artificial Neural Network (AAN) & $\begin{array}{c}\text { A mathematical statistical model imitating the } \\
\text { human brain in processing data and creating } \\
\text { patterns used in a decision making process [16] }\end{array}$ \\
\hline Machine Learning (ML) & $\begin{array}{c}\text { A subset of AI which learns from experience without } \\
\text { being explicitly programmed in order to deliver } \\
\text { specific outputs [17] }\end{array}$ \\
Deep Learning (DL) & $\begin{array}{c}\text { A subset of ML structured similar to the human } \\
\text { brain processing, using large multiple data sets at } \\
\text { the same time, evaluating and reprocessing multiple } \\
\text { time to reach an output [18] }\end{array}$ \\
\hline Convolutional Neural Network (CNN) & $\begin{array}{c}\text { An AAN that is particularly efficient when applied } \\
\text { to digitized images and pattern recognition [19] }\end{array}$ \\
\hline
\end{tabular}

This review focuses on the analysis of the last five years' published papers on AI and ML techniques by scrutinizing PubMed, Web of Science and Science Direct, using prostate cancer, biomarker, genomics, artificial intelligence and artificial neural networks keywords. We had also included the AI leading studies from later years or data on other diseases. The goal was to give an overview of the current evidence and future directions of AI in all the clinical management of prostate cancer patients from the diagnosis to the treatment.

\section{AI in Digital Pathology of Prostate Cancer}

\subsection{Developments in Optical Image Analysis}

Since 2010, Pantanowitz et al. [20] have talked about digital imaging and digital imaging processing. Digital whole histopathological slides are more interactive, easy to share, involve less preparation time and generate teaching sets (virtual slide boxes). Telepathology and image analysis by ML, using several algorithms such as computer assisted image analysis is something that has greater precision than the traditional microscope. The whole slide imaging, such as slide preservation over time, new handling of images, telepathology, quality assurance, education and collaborative research make the ML more easily understandable [21]. With the help of an augmented optical light microscope that enables the real-time integration of AI, Chen et al. [22] developed and evaluated deep learning algorithms for the fast identification of PCa. Further studies are needed to optimize the process.

\subsection{Differentiation of Cellular Structures and Tissues}

Nevertheless, quantitative morphometric features coming from image analysis may play a pivotal role in the diagnosis of PCa [23]. Some of the features have been included in automatic classifiers to differentiate stroma, normal glands and malignant tissue in PCa. With access to large sets of digitized tissue images from tissue microarrays (TMA), at the National Institutes of Health, Kwak et al. [24] used five different methods to identify cellular structures and the performance of the multi-view boosting methods. For the differentiation of cancer from benign tissue, the multi-view boosting classification showed a significantly higher AUC (area under the curve) (0.98 95\% CI 0.97-0.99) compared to the single-view classifications ( $p$-value $<0.01$ ) and outperformed the single view approach, which will increase the accuracy, robustness and utility of digital pathology tools analysis of tissues. In an observational study, Arvaniti et al. [25], on a training dataset of TMAs from 641 patients, small image patches extracted from benign tissue and PCa annotated regions were used to train a patch-based classifier. The stratification achieved by the authors' deep learning patch model, separated the low-risk and intermediate-risk groups significantly greater than 
the one achieved by either pathologist (Benjamini-Hochberg-BH-corrected two-sample log rank $p$-value $=0.098)$, respectively $(\mathrm{BH}$-corrected two-sample log rank $p$-values $=0.79$ and 0.292) and the model revealed a close inter-observer agreement (with kappa $=0.71$ and $0.75)$ which was seen between two ground truth pathologists (kappa $=0.71)$.

\subsection{Demographic and Histopathology Reports Data}

Roffman et al. [26] developed and validated a multiparametric ANN for PCa risk prediction and stratification. Based on clinical and demographic characteristics, the prehistopathological status allowed the model to predict PCa risk. The ANN model yielded high specificity $(89.4 \%)$ and low sensitivity $(23.2 \%)$ for the prediction of prostate cancer risk. Lenain et al. [27], enlisting histopathological data from 4470 PCa patients, used different machine learning approaches to analyze the staging information for $\mathrm{T}$ (tumor), $\mathrm{N}$ (nodes) and M(metastasis), such as support vector machines (SVM), random forest (RF), and extreme gradient boosting (XGB). They used for analyzing the ML model performance model, the precision, recall and F-Score a measure of a model's accuracy on a dataset [28], as the metrics and obtained classification of pathology best result for N (F1-Score 0.98) and M (F1-Score 0.99).

\subsection{Whole Slide Image Analysis in Prostate Biopsies (Cancer Detection and Grading) and Performance Comparison of AI Models and Pathologists}

Ström et al. [29] digitized 6682 slides from needle core biopsies of 976 patients and 271 from 93 men outside the study, then trained a DNN, evaluating the prediction of its presence, its extent and Gleason grade of cancer. The correlation between model and assigned pathologist was AUC 0.96 to detect cancer and a mean pair wise kappa 0.62 for assigning Gleason grades. Litjens et al. [30], with the data from 225 glass slides of PCa biopsies, trained a deep learning $\mathrm{CNN}$, a network that can be applied to every pixel in a whole slide image, this in order to detect PCa in the biopsy specimens, with an AUC for the 90th percentile analysis of 0.98 in slide detection cancer. Campanella et al. [31] evaluated the framework of whole slide images for prostate, with the goal of avoiding expensive and time-consuming pixel-wise manual annotations, obtaining best results on the prostate dataset $(\mathrm{N}=24,859)$ with an AUC of 0.989 at 20 times optical magnification and the automated removal of $75 \%$ of slides resulted in no loss in sensitivity. One year before, Campanella et al. [32], using 12,160 whole slide images, 2424 positive and 9736 negative, trained on the full dataset an AlexNet and a ResNet18 network, and pretrained on various image models on ImageNet, achieved results with their best model on the ResNet34 and VGG11-BN of 0.976 and 0.977 AUC, respectively. In PCa classification from histopathologic images, patch-wise cross-validation and single pathologist lead to biases, therefore using patient based cross-validation and the opinion of several experts [33,34], because it is well known that the PCa grading, which is variable with the experience of urologic pathologist [35], will lead to the better performance of classification methods. Nagpal et al. [36], trained a DL system on 1557 slides, and compared it to a reference standard provided by 29 pathology experts (mean accuracy was 0.61 on the validation dataset), and the model reached a higher diagnostic accuracy of $0.70(p=0.002)$, and is better leaning for patient stratification risk. A convolutional network analysis (CNN) by Lucas et al. [37] shows that, with proper training, the CNN can differentiate areas that are not atypical and malignant areas with an accuracy of $92 \%$, with a sensitivity and specificity of $90 \%$ and $93 \%$, respectively. Lately, Raciti et al. [38] published the results of how an AI system like Paige Prostate Alpha can influence pathologists during the diagnosis of PCa on biopsy needle cores. In diagnosing PCa with Paige Prostate Alpha, sensitivity for all pathologists increased with the AI system (average sensitivity without Paige Prostate Alpha: $74 \% \pm 11 \%$; with Paige Prostate Alpha: $90 \% \pm 4 \%$ ). To determine the Gleason grade groups, they found an increase in average sensitivity with a Paige Prostate Alpha of $20 \%$ for Grade group 1, 13\% for Grade group 2 and $11 \%$ for Grade group 3, allowing the pathologist to better classify lower grade groups. 
A population-based, diagnostic study aimed to identify PCa by an AI system, training deep neural network (DNN) can lead to better identification of the malignant from the benign cores of prostate biopsies (AUC 0.997(95\% CI 0.994-0.999) for the independent test dataset $($ benign $=910$, malignant $=721)$ and $0.986(0.972-0.996)$ for the external validation dataset (benign $=108$, malignant $=222$ ) and further fine tuning of the AI system will allow for better stratifying Gleason grading groups because they identified that the performance dropped in cancer length predictions and overall Gleason grading [29].

In another large trial published by Bulten et al. [39], they analyzed 5759 PCa core biopsies from 1243 patients and trained the deep-learning system to detect Gleason grade. In the test dataset, the deep-learning system achieved an AUC of 0.990 on determining the malignancy of a biopsy, on the observer set an AUC of 0.984 and the system outperformed 10 out of 15 pathologists and achieved the same results as the reference standard both for Grade group 2 or more (AUC 0.978, 0.966-0.988), and a grade group of 3 or more (AUC $0.974,0.962-0.984$ ). There are also limitations to the system because pathologists can assess the volume more qualitatively and the system counts the exact area of the individual glands and the findings of Egevad et al. [40] should be used to improve calibration of AI systems. Therefore, Bulten et al. [41] published yet other results showing that the limitations can be addressed if $\mathrm{AI}$ is assisting pathologists rather than having a competition in assessing the performance of either pathologists or AI systems. The list of studies discussing different types of algorithms used to train the models, which were looking into Gleason grading of biopsies, TMAs, whole section, the amount of data used and their results are listed in Table 2.

In summary, all evidence up to this moment shows that the AI systems are in need of further development to help and assist pathologists to provide an accurate diagnosis. AI could provide in the future the necessary aid in histopathological diagnosis, especially in remote areas and health systems that need the expertise of highly trained pathologists. Before any clinical setting usage of such systems they will have to be approved by health regulators. The quest for personalized approach in $\mathrm{PCa}$, the quantitative histopathological diagnosis will have to provide pathologist with new tools to increase the sensitivity and specificity of more accurate readings of the tissue images and AI seems to provide this better and faster. 
Table 2. AI studies, type of training in accordance to Gleason grading of biopsies, tissue microarrays (TMAs) and whole section and their results

\begin{tabular}{|c|c|c|c|c|c|}
\hline Article/Reference & Image Type & Image Analysis Method & $\begin{array}{l}\text { Number of Slides or } \\
\text { Patients (N) }\end{array}$ & Task & Results \\
\hline Campanella et al. [31] & Whole slide images & $\begin{array}{l}\text { Multiple instances learning } \\
\text { based, deep learning }\end{array}$ & $\mathrm{N}=24,859$ slides & Automated cancer detection & Model achieved AUC over 0.98 \\
\hline Campanella et al. [32] & Whole slide images & $\begin{array}{l}\text { Multiple instances learning } \\
\text { based, deep learning }\end{array}$ & $\mathrm{N}=12,160$ slides & Automated cancer detection & $\begin{array}{l}\text { Modelachieved an AUC of } 0.98 \text { and a false negative } \\
\text { rate of } 4.8 \%\end{array}$ \\
\hline Litjens et al. [30] & Whole slide images & Deep learning, CNN & $\mathrm{N}=225$ slides & Automated cancer detection & $\begin{array}{l}\text { AUC for the 90th percentile analysis of } 0.98 \text { in slide } \\
\text { detection cancer }\end{array}$ \\
\hline Arvaniti et al. [25] & Tissue microarrays & Deep learning, CNN & $\mathrm{N}=641$ patients & Automated Gleason grading & $\begin{array}{l}\text { Model revealed a close inter-observer agreement (with } \\
\text { kappa }=0.71 \text { and } 0.75) \text { which was seen between two } \\
\text { ground truth pathologists (kappa }=0.71) .\end{array}$ \\
\hline Lenain et al. [27] & Words analization & Natural language processing & $\mathrm{N}=4470$ patients & Classify free-text pathology reports & $\begin{array}{l}\text { Classification of pathology best result for } \mathrm{N} \\
\text { (F1-Score 0.98) and M (F1-Score 0.99) }\end{array}$ \\
\hline Ström et al. [29] & Whole slide images & Deep learning, CNN & $\mathrm{N}=6682$ slides & $\begin{array}{l}\text { Automated cancer detection and } \\
\text { Gleason grading }\end{array}$ & $\begin{array}{c}\text { Correlation between model and assigned pathologist } \\
\text { was AUC } 0.96 \text { to detect cancer and a mean pair wise } \\
\text { kappa } 0.62 \text { for assigning Gleason grades }\end{array}$ \\
\hline Nagpal et al. [36] & Whole slide images & Deep learning, CNN & $\mathrm{N}=1557$ slides & Automated Gleason grading & $\begin{array}{l}\text { Model achieved accuracy of } 0.70 \text { compared to } 29 \\
\text { pathologists }(0.61) \text { on the validation set, } p=0.002\end{array}$ \\
\hline Raciti et al. [38] & Whole slide images & $\begin{array}{l}\text { Paige Prostate Alpha Deep } \\
\text { learning, CNN }\end{array}$ & $\mathrm{N}=304$ slides & $\begin{array}{l}\text { Automated cancer detection and } \\
\text { Gleason grading }\end{array}$ & $\begin{array}{l}\text { Diagnosed PCa with Paige Prostate Alpha, sensitivity } \\
\text { for all pathologists increased with the AI system } \\
\text { (average sensitivity without Paige Prostate Alpha: } \\
74 \% \pm 11 \% \text {; with Paige Prostate Alpha: } 90 \% \pm 4 \% \text { ). } \\
\text { An increase in average sensitivity with Paige Prostate } \\
\text { Alpha, of } 20 \% \text { for Grade group 1, 13\% for Grade group } \\
2 \text { and } 11 \% \text { for Grade group } 3 \\
\end{array}$ \\
\hline Bulten et al. [39] & Whole slide images & Deep learning, CNN & $\mathrm{N}=1243$ patients & Automated Gleason grading & $\begin{array}{c}\text { Model achieved the same results as the reference } \\
\text { standard both for Grade group } 2 \text { or more (AUC 0.978, } \\
0.966-0.988 \text { ), and grade group of } 3 \text { or more (AUC } \\
0.974,0.962-0.984 \text { ) }\end{array}$ \\
\hline
\end{tabular}

Abbreviations: AUC—area under the curve, Abbreviations: CNN—convolutional neural network, kappa—Cohen's quadratic kappa statistic, AI—artificial intelligence, PCa—prostate cancer. 


\section{AI in Diagnostic Imaging of Prostate Cancer}

\subsection{ML in MRI Imaging Tools in PCa}

Early attempts to fusion pathology with imaging have been performed since 1991 by Schnall et al. [42], with limited success to correlate radiographic and pathological features. In 2012, Ward et al. [43] evaluated a technique to perform digital registration of images from histopathology and in vivo MRI using image-guided specimen slicing based on strand-shaped fiducial markers. Since 2014, Litjens et al. [44] have used segmentation and characterization of MRI images using several models (analyzing digital prostate images, pixels for basic image analysis) represents the base for the more recently discovered and applied methods. Segmentation of prostate is very important for identifying its deformable capsule, has application in prostate fusion biopsy, brachytherapy and can be done with the help of MRI and transrectal ultrasonography (TRUS). In a multi-institutional study, Gaur et al. [45] have shown that artificial intelligence based detection, improved specificity combined with PI-RADS (Prostate Imaging-Reporting and Data system) v.2 (version 2) categorization and they found a sensitivity for the described images for PIRADSv. $2 \geq 3$ of $78 \%$. Here, the greatest benefit was seen in the transitional zone (TZ), where it helped moderately experienced readers to achieve the level of the well experienced radiologists and help to improve the reading of the transitional zone was of $83.8 \%$ sensitivity with automated detection versus $66.9 \%$ with MRI alone [45].

Abdollahi et al. [46] used radiomics based models on T2 weighted images (T2W) that were more reliable than apparent diffusing coefficient (ADC) in MRI data, as apparent diffusing coefficient for Gleason score for staging of the disease seems to perform better. Lately, Dulhanty et al. [47] have used novel radiomics with ADC and computed high-b value diffusion weighted imaging (CHB-DWI) modalities for prostate cancer diagnosis with a better performance than a clinical heuristics driven strategy. Another DL based approach, presented by Aldoj et al. [48], a CNN using different 3D combinations (ADC, diffusion weighted imaging (DWI), T2 weighted images) with an AUC of 0.91 at $81.2 \%$ sensitivity and $90.5 \%$ specificity, compared to a radiologist using PI-RADS v2 [49].

The two PROSTATEx Challenges were an effort to improve, with the help of CAD, the classification of clinically significant $\mathrm{PCa}$ and the characterization of the Gleason grade group. The PROSTATEx Challenge involved quantitative image analysis methods to analyze prostatic lesions, and the PROSTATEx-2 involved quantitative MRI biomarkers for the determination of Gleason grade group in PCa [50]. As time passed, in 2020, de Vente et al. [51] characterized another computerized model, involving deep learning regression, in bi-parametric MRI examination, soft-label ordinal regression improves the performance of PCa grading and detection from biparametric-MRI over earlier presented methods. A retrospective analysis [52], using the data collected in 2017 from the PROSTATEx Challenges, trained a CNN model and the best input achieved in this study was a combination of T2-weighted images, ADC and DWI, and the results were that an end-to-end training of the CNN model, with data from different scanners and protocols, can be generalized and more validation in the future is needed. To minimize the interference of different MRI scanners and image acquisition protocols, Sunoqrot et al. [53] proposed a model that uses automatic (dual fat and muscle reference approach), signal intensity normalization to improve T2-weighted MR images of the prostate using object recognition, and significantly higher AUC (0.826 vs. 0.769) for the classification of histologically diagnosed peripheral zone tissues compared to the other methods. Because of the variation between diagnoses of different radiologist using PIRADS for assessing prostate lesions, a DL was developed further to help with the characterization of clinically significant $\mathrm{PCa}$, and the results were that the CNN trained has the similar power as an experienced radiologist [54]. New MRI techniques are developed to improve the quality and acquisition time of image sequences, quantitative imaging, computer-aided diagnosis and artificial intelligence (luminal water imaging -LWI, Restriction spectrum imaging-RSI, vascular, extracellular and restricted diffusion for cytometry in tumors-VERDICT, hybrid multi-dimensional MRI-(HM-MRI), MR fingerprinting (MRF), segmented or multi-shot DWI), to better detect, characterize, 
diagnose and predict prognoses for PCa [55]. Many of the examples of algorithms used have to train to define each zone that is in a spatial label on MRI, to be in accordance with the Gleason score of each tumor. For sure, spatial annotation at full resolution of the digital histopathological images will improve the ability of the machine learning techniques to differentiate normal versus malignant tissues [56].

\section{2. $M L$ in TRUS Imaging in PCa}

Karimi et al. [33] identified a model that means patch-based training and evaluation could lead to significant overestimation of a model's predictive accuracy. The importance is that patient-based training and evaluation is the only acceptable method for developing machine learning models in this application. The DL network is trained to extract and to learn its own features on the basis of the raw image to improve the classification of the image compared with the ML approach [7].

AI can help by decreasing the time of reader that interprets the data; therefore, there will be an increase in the performance of radiologists. Applications of AI to prostate MRI can specifically increase the sensitivity of PCa detection and decrease inter-reader variability [57].

Compared to the clinical TRUS segmentation, the Zeng at al. [58] method that used the CNN statistical shape model was able to achieve lower volume matching results in all prostate regions, but especially at the base and apex. Karimi et al. [59] proposed a new CNN architecture for prostate clinical target volume segmentation in TRUS images, which computes multi-scale features directly from the input images, which learns similarities in all training images, then cross-validated them, and developed a method to improve uncertain segmentations based on the estimated uncertainty map and the expected shape. Feng et al. [60] proposed a new method, which extracts features from both the spatial and the temporal dimensions by performing three-dimensional convolution operations and when compared to other methods this method achieved a sensitivity of $82.98 \pm 6.23$, a specificity of $91.45 \pm 6.75$ and an accuracy of $90.18 \pm 6.62$ in PCa detection using contrast enhanced ultrasonography (CEUS), anti-PSMA (prostate specific membrane antigen) and the nontargeted blank agent as contrast agents. Wildeboer et al. [61] assessed the potential of ML B-mode, shear-wave elastography (SWE) and dynamic contrast-enhanced ultrasound (DCE-US) with a high result compared to contrast velocity with an AUC of 0.75 and 0.90 for PCa and Gleason $>3+4$.

\subsection{Combined MRI and TRUS for ML in Prostate Cancer}

Fusion biopsy relies on distant past frames and newly acquired in real time TRUS and proper segmentation using MRI and TRUS. Moving from state-of-the art techniques that use the deep CNN in visual recognition tasks and that have superior performance in obtaining reliable images for prostate mapping in brachytherapy techniques [62], Anas et al. [63] proposed an automatic prostate segmentation technique that incorporates temporal information of TRUS images to improve the segmentation accuracy and has the ability to enable real-time deformable registration and improved biopsy guidance. A CNN was proposed for the registration of T2-weighted MRI and 3D TRUS volumes of the prostate [64] and then a hybrid 3D/2D U-Net CNN (the Hybrid 3D/2D U-Net was trained on 3D images and then completed object detection and segmentation on 2D images) approach to prostate organ segmentation was described as having good performance in regard to prostate segmentation and volumetric evaluation [65]. Liu et al. [66] recruited 50 confirmed PCa patients with a Prostate Imaging-Reporting and Data System version 2 (PI-RADS v2) score of 4 or 5. Logistic regression based on the first and strongest enhancement phase (Dataset-FS) integration; enhanced phases of dynamic contrast enhanced magnetic resonance imaging (DCE-MRI) can lead to the diagnostic ability of the model to predict PCa invasiveness with a noninvasive accuracy of 0.90. Ishioka et al.'s [67] model of computer-aided diagnosis, based on a CNN algorithm combined with U-net with ResNet50, showed AUC values in the two evaluation data sets, 0.645 and, respectively, 0.636 , for estimating the designated 
area in which targeted biopsy confirmed the presence of PCa, and AUC values had improved as the DL progressed and they found an improvement in diagnostic accuracy by lowering the number of patients mistakenly diagnosed as having cancer. The accuracy of an imaging diagnosis and the improvements related to developments in DL techniques could further lower the inter and intra-observer variability and make faster learning curves in reading PCa scoring systems such as PI-RADS. A summary of the included studies and their different imaging used modalities, combinations and techniques, artificial intelligence used methods and their performance are listed in Table 3.

Table 3. Different imaging modalities and techniques, AI method and performance and combination of imaging modalities.

\begin{tabular}{|c|c|c|c|c|}
\hline Article/Reference & $\begin{array}{l}\text { Imaging } \\
\text { Modality }\end{array}$ & Imaging Technique & AI Method & Performance \\
\hline Litjens et al. [44] & MRI & T2W, PD, DWI, DCE & RFC & AUC 0.89 \\
\hline Gaur et al. [45] & MRI & T2W, DWI, DCE, ADC & RFC & $\begin{array}{l}\text { Benefit in TZ for moderately-experienced readers at } \\
\text { PI-RADSv } 2<3(84 \% \text { vs mpMRI-alone } 67 \%, p=0.055) \text {, } \\
\text { and PI-RADSv2 } \geq 3 \text {, CAD improved patient-level } \\
\text { specificity (72\%) compared to mpMRI-alone (45\%, } \\
\qquad p<0.001) \text {. }\end{array}$ \\
\hline Zeng et al. [58] & $\begin{array}{l}\text { MRI, } \\
\text { TRUS }\end{array}$ & T2W, B-mode & $\mathrm{CNN}$ & $\begin{array}{l}\text { Reduction in the segmentation error at base and apex, } \\
5-10 \% \text { reduction in aRVD. }\end{array}$ \\
\hline Karimi et al. [59] & TRUS & B-mode & $\mathrm{CNN}$ & $\begin{array}{l}\text { CNN method outperformed other methods in DSC, } \\
\text { HD } p=0.01\end{array}$ \\
\hline Feng et al. [60] & TRUS & CEUS & $\mathrm{CNN}$ & $\begin{array}{c}\text { Sensitivity of } 82.98 \pm 6.23, \text { specificity of } 91.45 \pm 6.75 \\
\text { and accuracy of } 90.18 \pm 6.62 \text { compared to } \\
\text { other methods }\end{array}$ \\
\hline Wildeboer et al. [61] & TRUS & B-mode, SWE, CEUS & RFC & $\begin{array}{l}\text { AUC } 0.90 \text { for PCa and Gleason }>3+4 \text {, outperforming } \\
\text { contrast velocity }\end{array}$ \\
\hline Hu et al. [64] & $\begin{array}{l}\text { MRI, } \\
\text { TRUS }\end{array}$ & T2W, 3D B-mode & GMM & $\begin{array}{l}\text { Median target registration error of } 3.6 \mathrm{~mm} \text { on } \\
\text { landmark centroids and a median Dice of } 0.87 \text { on } \\
\text { prostate glands }\end{array}$ \\
\hline Liu et al. [66] & MRI & T1W, T2W, DWI, DCE & SVM, RF, RFC, DT, KNN & $\begin{array}{l}\text { KNN—best predict efficacy } \\
\text { AUC- } 0.88 \text {; accuracy- } 0.85\end{array}$ \\
\hline Ishioka et al. [67] & MRI & $\mathrm{T} 2 \mathrm{~W}$ & $\mathrm{CNN}$ & $\begin{array}{l}\text { AUC } 0.645 \text { estimating the designated area in which } \\
\text { targeted biopsy confirmed the presence of } \mathrm{PCa}\end{array}$ \\
\hline Abdollahi et al. [46] & MRI & T2W, ADC & $\mathrm{CNN}$ & $\begin{array}{l}\text { For GS prediction, T2 } \mathrm{W} \text { radiomic models more } \\
\text { predictive (mean AUC 0.739) than ADC models } \\
\text { (mean AUC 0.70). For stage prediction, ADC models } \\
\text { higher prediction performance (mean AUC 0.675) }\end{array}$ \\
\hline Aldoj et al. [48] & MRI & T2W, ADC, DWI & $\mathrm{CNN}$ & $\begin{array}{l}\text { AUC of } 0.91 \text { at } 81.2 \% \text { sensitivity and } 90.5 \% \text { specificity, } \\
\text { compared to radiologist using PI-RADS v2 }\end{array}$ \\
\hline de Vente et al. [51] & MRI & T2W, ADC, DWI & $\mathrm{CNN}$ & $\begin{array}{l}\text { Voxel-wise weighted kappa of } 0.446 \pm 0.082 \text { and a } \\
\text { Dice similarity coefficient for segmenting clinically } \\
\text { significant cancer of } 0.370 \pm 0.046 \text {, above ProstateX-2 }\end{array}$ \\
\hline Sunoqrot et al. [53] & MRI & $\mathrm{T} 2 \mathrm{~W}$ & $\mathrm{CNN}$ & $\begin{array}{l}\text { Healthy vs. malignant classification also improved } \\
\text { significantly ( } p<0.001 \text { ) in peripheral (AUC } 0.826 \text { vs. } \\
0.769 \text { ) and transition (AUC } 0.743 \text { vs. } 0.678 \text { ) zones. }\end{array}$ \\
\hline
\end{tabular}

Abbreviations: RFC—random forest classifiers, DSC—dice similarity coefficient, HD—Hausdorff Distance, GMM—gaussian mixture model, CEUS — contrast enhanced ultrasonography, PD—proton density imaging, SWE-shear wave elastography, ADC—apparent diffusing coefficient, T2W-T2 weighted imaging, T1W—T1 weighted imaging, DWI—diffusion weighted imaging, DCE—dynamic enhanced contrast imaging, SVM—support vector machine, RF—random forest, DT—decision tree, KNN—K-nearest neighbor.

\section{AI in Prostate Cancer Genomics}

In order to predict individual outcomes in patients with $\mathrm{PCa}$, there is an increased interest in the genomics of PCa and how the alterations in the PCa genome can change the individual evolution of his PCa [68]. In the last five years there has been a scarcity of scientific data in terms of genetic research, with an increase in the last few years. 


\subsection{Approach in mRNA, miRNA and SNPs (Single Nucleotide Polymorphisms)}

A few data are available from 2016, when Bertoli et al. [69], using a meta-analysis approach, identified through an ML approach a group of 29 miRNAs that can be used for diagnostic purposes and a group of 7 miRNAs that may have prognostic abilities. MacInnis et al. used a novel analysis method, back in 2016, with the dependency of association on the number of Top Hits, that identified 14 regions associated with PCa using a conventional logistic regression analysis of individual single-nucleotide polymorphisms [70]. Decipher uses a random forest algorithm to predict PCa metastatic disease [7,71]. Another study of Lee et al. [72] used ML (pre-conditioned random forest regression) and bioinformatics tools to assess, on genome based study, the conditions that appear after radiotherapy and to predict late toxicity, resulting in a statistically significant prediction model $(p=0.01)$, only for weak stream. DNA methylation markers have been identified as having diagnostic ability.

\subsection{ML in Gene Expression and Gene Activity}

Hou et al. [73] used a genetic algorithm optimized artificial neural network to establish a diagnostic model that showed good results for the diagnosis $(\mathrm{AUC}=0.953)$ and prognosis (AUC of 5 years overall survival time $=0.808$ ) of PCa. Liu et al. [74] identified $12 \mathrm{CpG}$ (cytosine and guanine on a genome) site markers and 13 promoter markers, using a deep neural network model, from an initial pool of 139,422 CpG sites, and the promoter methylation data contained 15,316 promoters and applied three machine learning strategies (moderated t-statistics, LASSO, and random-forest).This might further be used for liquid biopsy of cancers. Lately, Hamzeh et al. [75] used a combination of efficient machine learning methods (Support Vector Machine (SVM)-Radial basis function kernel (SVM-RBF), Naive Bayes, Random Forest) to analyze gene activity and to identify the genes for the presence of PCa (on one side or both sides). The highest accuracy and precision for the different classifiers came from the SVM-RBF classifier, which was able to separate the different locations by an accuracy of $99 \%$ and found genes (HLA-DMB and EIF4G2) that are correlated with PCa progression. de la Calle et al. [76] aimed to predict the recurrence and progression of PCa based on biomarker analysis from 648 samples (424 tumors, 224 normal tissue) using tissue micro assays anti Ki-67, anti ERG (erythroblast transformation-specific related gene) antibodies through an $\mathrm{AI}$ algorithm, having $100 \%$ identification of ERG positive tumors. Genomics is playing an important role in the outcome of PCa patients. Genes could be identified through ML as candidate biomarkers or with a potential diagnostic role but for the future, great computational power will be required to increase the receiver operating characteristics in prognostic for the individual patient, through fused data streams. At this point, the scarcity of studies to report on fused data streams is linked to the challenges with the study of genomics. Further studies linked to data fused streams are required to identify the best ML methods, or to improve the existing ones, to tackle the challenges in clinical application of AI in genomics. A summarization of the discussed studies are imbedded in Table 4.

Table 4. AI and machine learning (ML) techniques used to identify alterations in human genome correlating in prostate cancer (PCa), usefulness radiotherapy and in prostate cancer surgery.

\begin{tabular}{|c|c|c|c|}
\hline Genomics Article/Reference & Genomics Analized/Image Feature & AI Method & Performance \\
\hline Bertoli et al. [69] & miRNA & SVM & $\begin{array}{c}\text { Diagnostic } 29 \text { miRNA AUC } 0.989 \pm 0.016 \\
\text { Prognostic signatures } 7 \text { miRNA best AUC } \\
74.7 \% \text { (CI 95\%): } 73.28-76.11\end{array}$ \\
\hline Karnes et al. [71] & mRNA expression, 22 genes & $\mathrm{RF}$ & $\begin{array}{c}\text { AUC } 0.79,5 \text { years metastasis free survival after } \\
\text { surgery }\end{array}$ \\
\hline Lee et al. [72] & Single nucleotide polymorphisms & $\begin{array}{l}\text { Preconditioned random forest } \\
\text { regression }\end{array}$ & AUC $0.70($ CI95\%, $0.54-0.86, p=0.01)$ \\
\hline
\end{tabular}


Table 4. Cont.

\begin{tabular}{|c|c|c|c|}
\hline Genomics Article/Reference & Genomics Analized/Image Feature & AI Method & Performance \\
\hline Hou et al. [73] & Gene expression & GA-AAN & $\begin{array}{c}\text { AUC } 0.953 \text { for diagnosis } \\
\text { AUC } 0.808 \text { for prognosis ( } 5 \text { year overall } \\
\text { survival) }\end{array}$ \\
\hline Liu et al. [74] & DNA methylation markers & $\begin{array}{c}\mathrm{CNN} \text {, moderated t-statistics, LASSO, } \\
\text { and RF }\end{array}$ & $\begin{array}{c}\text { CpG markers } 100 \% \text { sensitivity and promoter } \\
\text { markers } 92 \%\end{array}$ \\
\hline Hamzeh et al. [75] & Gene activity & $\begin{array}{l}\text { SVM-Radial basis function kernel- } \\
\text { SVM-RBF, Naive Bayes, RF }\end{array}$ & $\begin{array}{l}\text { Highest accuracy and precision: SVM-RBF } \\
\text { classifier } \\
\text { Accuracy } 99 \% \\
\text { HLA-DMB and EIF4G2 correlated with PCa } \\
\text { progression }\end{array}$ \\
\hline Shiradkar et al. [77] & $\begin{array}{l}\text { Radiomics MRI based } \\
\text { Multimodal co-registration scheme to } \\
\text { map the prostate } \\
\text { Radiomics based dose plan on MRI for } \\
\text { brachytherapy and on CT for EBRT }\end{array}$ & Machine learning classifier- QDA & $\begin{array}{l}\text { Reduction in dosage in radiomics based focal } \\
\text { therapy compared to whole gland in EBRT and } \\
\text { brachytherapy }\end{array}$ \\
\hline Lei et al. [78] & TRUS 3D V-Net & $\begin{array}{c}\text { CNN } \\
\text { Deeply supervised V-Net }\end{array}$ & $\begin{array}{c}\text { DSC } 0.92 \pm 0.03 \\
\text { HD } 0.94 \pm 1.55 \mathrm{~mm} \\
\text { MSD } 0.60 \pm 0.23 \mathrm{~mm} \\
\text { RMSD } 0.90 \pm 0.38 \mathrm{~mm}\end{array}$ \\
\hline Nouranian et al. [79] & TRUS, CTV, PTV & $\begin{array}{l}\text { Joint sparse dictionary } \\
\text { learning approach }\end{array}$ & $\begin{array}{c}\text { Correlation between CTV and PTV }=16.28 \pm \\
2.39 \% \mathrm{~V} \text { err in estimation of PTV from CTV } \\
\text { (single label model (TRUS } / \text { PTV) higher error vs } \\
\text { multilable approach }(p<0.01)\end{array}$ \\
\hline Karimi et al. [59] & TRUS & $\mathrm{CNN}$ & $\begin{aligned} \mathrm{DSC} & =93.9+/-3.5 \%, \mathrm{HD}=2.7 \pm 2.3 \mathrm{~mm} \\
(p & =0.01 \text { compared to other methods })\end{aligned}$ \\
\hline Nicolae et al. [80] & LDR treatment plan & $\mathrm{CNN}$ & $\begin{array}{l}\text { Planning time for the ML algorithm }=0.84 \pm \\
0.57 \text { min compared to } 17.88 \pm 8.76 \text { min for the } \\
\text { expert planner }(p=0.020) \\
\text { Pre-implant plans were dosimetrically } \\
\text { equivalent to the BT plans; the average prostate } \\
\text { V150\% was } 4 \% \text { lower for ML plans }(p=0.002) ;\end{array}$ \\
\hline Sanders et al. [81] & $\begin{array}{l}\text { Seed localization performance } \\
=\text { computing the RMSE }\end{array}$ & Sliding-window CNN algorithm & Slightly increased the run-time \\
\hline Surgery Article/Reference & Image Features & AI Method & Performance \\
\hline Sarikaya et al. [82] & $\begin{array}{l}\text { Instrument detection and localization } \\
\text { in robotic assisted surgery images }\end{array}$ & CNNEnd-to-end deep learning & $\begin{array}{l}\text { Improves the accuracy and reduces the } \\
\text { computation time for detection in each frame } \\
\text { AP }=91 \% \\
\text { Training time }=7.22 \mathrm{~h} \\
\text { Computation time }=0.103 \mathrm{~s} \text {, each frame }\end{array}$ \\
\hline Hung et al. [83] & Automated performance data & ML algorithms & Bimanual dexterity = an ideal surgical skill \\
\hline
\end{tabular}

Abbreviations: RF-random forest, GA-AAN-genetic algorithm artificial neural network, RNA-Ribonucleic acid, DNAdeoxyribonucleic acid, SVM — support vector machine, AUC - area under the curve, CNN—convolutional neural network, SVM-RBFRadial Basis Function Support Vector Machine, MRI-magnetic resonance imaging, CT—computed tomography, TRUS—-transrectal ultrasonography, QDA-Ribonucleic acid, EBRT—deoxyribonucleic acid, DSC-Dice Similarity Coefficient, HD-Hausdorff distance, MSD-Mean surface distance, RMSD—Residual mean surface distance, CTV—clinical target volume, PTV—planning target volume, ML-Machine Learning, BT—Brachytherapist, RMSE—root mean square error, AP—average precision.

\section{AI in Prostate Cancer Treatment}

\subsection{AI in Prostate Cancer Radiotherapy}

\subsubsection{MRI Based ML Approach for Treatment of PCa}

The artificial intelligence and machine learning techniques described for imaging different types of cancers can be extended to treatment planning that involves radiotherapy. For brachytherapy and external beam radiation therapy (EBRT), radiomics-based detection of cancerous patches described in MRI were transferred on to a computed tomography (CT) scan for EBRT, using a texture feature enabled machine learning classifier, to achieve a deformable map to accurately predict the cancer lesions [77]. For treatment planning, using a deep attention U-Net network that integrates attention gates and deep supervision, Dong et al. [84] compared models with or without deep attention algorithms. Compared to $\mathrm{CT}$, deep attention networks a synthetic MRI (sMRI), especially developed for soft tissues, which obtained better results in volume overlapping, better surface matching and better center and volume matching, probably offering better PCa radiotherapy treatment planning. Savenje et al. [85] investigated the feasibility of the clinical use of organs at risk of autosegmentation based on CNN DeepMedic and V-net, using MRI images and the qualitative 
analysis showed that delineation from DeepMedic required fewer adaptations and less time for the delineation procedure, therefore it is important for the optimization of clinical workflow. In an effort to investigate the accuracy of dose calculations in PCa radiotherapy, Shafai-Erfani et al. [86] used a CNN algorithm, random forest, from synthetic CT images generated from MRI images. Quantitative results showed no significant differences in dose volume histogram and planning target volumes, showing that in the future ML-MRI methods could generate synthetic CT images from MRI and could probably eliminate CT acquisition and eliminate its radiation toxicity.

\subsubsection{Approach in TRUS Studies for Treatment of PCa}

For brachytherapy, ultrasound studies on prostate segmentation, a deeply supervised deep learning-based approach [78], an efficient learning-based multi-label segmentation algorithm [79] and an estimation of model uncertainty and use of prior shape information to significantly improve the performance of CNN-based medical image segmentation methods [59], show clinical feasibility in terms of accuracy compared to manual segmentation of the prostate and potential benefit for clinical applications that can help clinicians for training and decision support for intraoperative planning or targeted biopsy. This includes from evaluating ML algorithms for treatment planning in prostate brachytherapy with expected improvement in prostate low dose rate treatment plans to lower planning time and resources [80], to the use of quantitative susceptibility mapping and unsupervised machine learning to accurate and robust manner to localize the radioactive seeds [87], to the implementation of a sliding-window convolutional neural network for radioactive seed identification in MRI-assisted radiosurgery. This is to improve seed identification [81] and the radiation dose calculations using CNN models to help improve the speed of those calculations [88-90]. The outcomes of toxicity following radiotherapy were assessed by Isaksson et al. [91] in a review of PCa radiotherapy treatment in terms of genitourinary and gastrointestinal toxicity, and the publications screened only a few that showed better performance than classical models. By adding more features when training the model (the use of statin drugs and PSA (Prostate Specific Antigen) level prior to intensity, modulated radiotherapy was found to be strongly related to the toxicity outcome. DL based methods will be able to calculate the radio-therapeutic dose with accuracy and efficiency and this will have an important role for real time radiotherapy. In the future, DL will be a valuable asset in many different aspects for both patients and clinicians. A summary of studies can be found in Table 4.

\subsection{AI in Prostate Cancer Surgery}

Robotic assisted platforms for prostate surgery (e.g., radical prostatectomy) already use artificial intelligence through machine learning based methods [92]. Some of the potential applications of ML in surgical robotics are automation of the surgical operation, saving the best strategies of high volume surgeons, training surgeons, classification and standardization of surgical procedures, safe interaction between environment and surgical robots and safe interaction between surgeons and surgical robots [93]. If we look into the future, automatizing the surgical procedure is a real desire. The complexity of an autonomous system that can perform surgical interventions is very high and there is a need to cover all aspects of a surgical procedure and to develop a system that can transfer the surgical skills toward automated execution. Sarikaya et al. [82] proposed in 2017 an end-toend deep learning approach for instrument detection and localization in robotic assisted surgery images. By using a CNN processing stream and multimodal convolutional network, they demonstrated that the proposed model is better than similar approaches, but the process is still slow in computing all images and could represent a basis for further studies. The research on how open radical prostatectomy and robotic assisted radical prostatectomy influences the emotions of patients in the two treatment groups was assessed with Patient Reported Information Multidimensional Exploration version2, from the online discussions of patients in different support groups to automated identification and intelligent analysis 
of emotions [94]. The biochemical recurrence was analyzed in patients following robotic radical assisted prostatectomy using three supervised ML algorithms and multiple training variables, with the result that ML techniques can produce accurate disease predictability in all three models, which is better than traditional statistical regression [95]. Hung at al. [83] used a da Vinci system recorder to collect automated performance data, adding them to ML algorithms, and found that bimanual dexterity is an ideal surgical skill and camera manipulation strongly correlates with surgeon expertise and good outcomes. Goldenberg at al. [7] developed a system that uses a computer controlled TRUS transducer in the rectum and tracks the surgical instrument tips and the real time MRI and TRUS images. These images enable the visualization of suspected lesions in real time. AI uses software for imaging in real time, intraoperative modifications and this will require a team effort between regulatory authorities and the research and development of manufacturers of the equipment to better implement the technology in the future, to provide better clinical outcomes for the patients. A list of analyzed papers is integrated in Table 4.

\section{Limitations and Future Perspectives}

The evidence at this time, highlighted in the present papers, points out that AI could advance pathology, imaging, genomics and surgery through how we understand AI and its advantages. Now AI is still developing and advancing. The time to train an AI system is high and it is not possible without human intervention. Some of the tasks of AI can surprisingly match, in some perspectives, the performance of experts but still limitations and challenges remain [41]. Regulation is mandatory for every test that will be introduced in clinical practice. The latest regulatory papers in the European Union and the United States of America will undergo certification beyond self-validation and certification studies to prove the reproducible result of the test and to avoid its nonreproducible risk [96,97].

One of the challenges is how these AI ML models will join clinical practice. How the leading researchers will put into practice a model that can predict $\mathrm{PCa}$, diagnosing it with histopathological or with imaging methods, remains a challenge [98].

It is estimated that in the future there will be commercially available tools to predict $\mathrm{PCa}$, or to grade PCa with the aid of AI. But presently, an AI method is limited by small data sets, and for the models to have a broader impact, they will have to have very large and representative data sets and images. Fine tuning a DL method, standardizing and controlling the process to improve the model, would further reduce errors through the generalization of a process. Some of the limitations that will have to be surpassed are related to the costs of digitalizing images, software and hardware acquisition, the need to show pathologists that AI is safe and can be applied to large cohorts and to set a threshold at which an AI model is performing at least as well as the pathologist, which is a multi-view approach to identifying cancerous tissue and differentiating it from benign tissue. Deep learning methods seem to be the most appropriate models to be applied in histopathology, especially through image data sets analysis and classification (amount of slides, pixels, image digitalization). For the future it will be very interesting to see whether, in image slide analysis of morphometric features, images will correlate with radiological methods and proteomics. For sure, future studies will show the extent of this possible association. DCNN or DNN is the state of the art method that had achieved great accuracy in terms of classification in medical imaging [29]. But present studies cannot recommend AI in pathology in a clinical setting. Algorithms used for radiological imaging are focused on registration, segmentation and radiomics, and the trend to automatization is on. The actual methods that were studied had shown little advancements on how to deliver the information to the physician. Most of the papers presented are focused on segmentation, acquisition of images, the quality of picture obtained and in the future, transfer adaptation techniques and supervision will be of great importance [99]. Currently, algorithms analyze the lesions and require manual training of the models. The current methods try to color code the lesions and transparency to low probabilities and are the most advanced in automatic visualization [100]. The targeted biopsy still poses a challenge in accuracy. Movements, 
prostate image deformation and poor alignment lead to errors. An integrated system that allows real-time imaging and biopsy will limit the underdiagnoses [101]. AI and deep learning techniques will have the ability to change the inter- and intra-observer variability and the limitations given by the interpretation of scoring systems, such as PIRADS, from less experienced physicians [99]. Further research is mandatory to obtain better prediction of malignancies in PCa images, to accurately biopsy those lesions and to properly diagnose clinically significant PCa.

The diagnosis and prognosis of PCa has been guided by PSA as a biomarker. In the last ten years there have been numerous biomarkers identified with potential use in clinical practice. Those biomarkers' potential for diagnoses and the prediction of prognosis will have to be integrated in a clinical setting. An ANN can play an important role in analyzing biomarkers such as KI-67 and ERG antibodies [14,76]. There is the potential that AI will provide fast and possibly more reliable identification and validation of biomarkers in PCa.

Radiotherapy and AI is based on the capacity of systems to provide better MRI and TRUS images with proper segmentation and shaping of organ boundaries in order to provide the therapeutic dose for prostate, in both EBRT and brachytherapy. There are studies that used ML to develop methods to better localize radioactive seeds [81,87], using CNNs to calculate the right dose. DL based methods will be able to calculate the radio-therapeutic dose with accuracy and efficiency in order to reduce toxicity [91]. AI techniques will need future studies to better identify anatomical regions for radiotherapy, better radioactive seed implantation to cover the lesions, and dose calculations to reduce radiotherapy related toxicity.

AI software is becoming more attractive, especially for ML algorithms in the construction of 3D models, which could be integrated in augmented reality and virtual reality systems for surgical purposes. Still, obtaining new technologies to perform automatic intraoperative image overlapping is an area of development [92]. An ML that will predict surgical movements, along with visualization in real time of the organ localization of tumors, is a field that needs further research and it could be of great use to surgeons in the future.

Overall, the AI methods trained to provide assistance to clinicians have to have clinical applications. They will have to be adapted to be easily understood and used by physicians. They will have to perform at least as well the experts in their fields of pathology, imaging, radiotherapy and surgery to be accepted by clinicians as of benefit to their patients. They will to be approved by regulatory agencies across the world and this will not be an easy task. There is a need for further studies to validate AI ML methods for clinical use.

\section{Conclusions}

In PCa, artificial intelligence and ANN algorithms (especially DCNNs) are promising for diagnosis and playing a predictive role in the prognoses of the disease. With sparse evidence, the need for further studies is real. The potential for diagnostic imaging, histopathology, genomics and treatment can hold great promise for the future and can improve the individualization of the disease and therefore improve patient outcomes in a more personalized fashion. The potential of AI in prostate cancer surgery will boost training and surgery performance for the future, both in terms of improvement outcomes for the patients, and also for the benefit of training and assessing surgical skills.

Author Contributions: Conceptualization, O.S.T. and M.D.V.; methodology, O.S.T., M.F.; software, O.S.T., M.F. and M.D.V.; validation, J.J.R., O.V. and G.L.; formal analysis, F.P., D.A., O.d.C., M.D.V. , M.F. and O.S.T.; resources, M.D.V.; data curation, O.S.T., M.M., G.C.; writing-original draft preparation, O.S.T.; writing — review and editing, O.S.T., M.F., D.T. and M.D.V.; supervision, U.F., D.T., O.d.C., G.M.B., F.D.G. and M.F.; project administration, O.S.T.; funding acquisition, M.D.V. All authors have read and agreed to the published version of the manuscript.

Funding: This work was supported by a grant of the Romanian Ministry of Education and Research, CNCS-UEFISCDI, project number PN-III-P1-1.1-PD-2019-0085, within PNCDI III.

Institutional Review Board Statement: Not applicable. 
Informed Consent Statement: Not applicable.

Conflicts of Interest: The authors declare no conflict of interest.

\section{Abbreviations}

\begin{tabular}{|c|c|}
\hline Acronyms & Description \\
\hline AAN & Artificial neural networks \\
\hline ADC & Apparent diffusing coefficient \\
\hline AI & Artificial intelligence \\
\hline anti-PSMA & Prostate specific membrane antigen \\
\hline AUC & Area under the curve \\
\hline B-mode & Brightness mode \\
\hline CAD & Computer aided diagnosis \\
\hline CDSSs & Clinical decision support systems \\
\hline CEUS & Contrast enhanced ultrasonography \\
\hline CEUS & Contrast enhanced ultrasonography \\
\hline CHB-DWI & Computed high-b value diffusion weighted imaging \\
\hline $\mathrm{CNN}$ & Convolutional neural network \\
\hline CpG & Cytosine and guanine on a genome \\
\hline $\mathrm{CT}$ & Computed Tomography \\
\hline Dataset-FS & First and strongest enhancement phase \\
\hline DCE & Dynamic contrast-enhanced \\
\hline DCE-US & Dynamic contrast-enhanced ultrasound \\
\hline DCNN & Deep convolutional neural network \\
\hline DL & Deep learning \\
\hline DNA & Deoxyribonucleic acid \\
\hline DT & Decision Tree \\
\hline DWI & Diffusion weighted imaging \\
\hline EBRT & External beam radiation therapy \\
\hline GA-AAN & Genetic algorithm artificial neural network \\
\hline Hybrid 3D/2D U-Net CNN & $\begin{array}{l}\text { The Hybrid 3D/2D U-Net trained on 3D images and then } \\
\text { completed object detection and segmentation on 2D images) }\end{array}$ \\
\hline KNN & K-nearest neighbor \\
\hline LASSO & Least absolute shrinkage and selection operator \\
\hline LWI & Luminal water imaging \\
\hline ML & Machine learning \\
\hline MRF & MR fingerprinting \\
\hline MRI & Magnetic resonance imaging \\
\hline $\mathrm{PCa}$ & Prostate cancer \\
\hline PI-RADS & Prostate Imaging-Reporting and Data system \\
\hline PSA & Prostate Specific Antigen \\
\hline RF & Random forest \\
\hline RFC & Random forest classifiers \\
\hline RNA & Ribonucleic acid \\
\hline RSI & Restriction spectrum imaging \\
\hline SVM & Support vector machines \\
\hline SVM-RBF & SVM-Radial basis function kernel \\
\hline SWE & Shear-wave elastography \\
\hline TMA & Tissue microarrays \\
\hline TRUS & Transrectal ultrasound \\
\hline $\mathrm{TZ}$ & Tranzitional zone \\
\hline XGB & Extreme gradient boosting \\
\hline
\end{tabular}




\section{References}

1. Bray, F.; Ferlay, J.; Soerjomataram, I.; Siegel, R.L.; Torre, L.A.; Jemal, A. Global Cancer Statistics 2018: GLOBOCAN Estimates of Incidence and Mortality Worldwide for 36 Cancers in 185 Countries. CA Cancer J. Clin. 2018, 68, 394-424. [CrossRef] [PubMed]

2. Siegel, R.L.; Miller, K.D.; Jemal, A. Cancer Statistics, 2020. CA Cancer J. Clin. 2020, 70, 7-30. [CrossRef] [PubMed]

3. Jović, S.; Miljković, M.; Ivanović, M.; Šaranović, M.; Arsić, M. Prostate Cancer Probability Prediction by Machine Learning Technique. Cancer Investig. 2017, 35, 647-651. [CrossRef]

4. Pang, B.; Zhu, Y.; Ni, J.; Thompson, J.; Malouf, D.; Bucci, J.; Graham, P.; Li, Y. Extracellular Vesicles: The next Generation of Biomarkers for Liquid Biopsy-Based Prostate Cancer Diagnosis. Theranostics 2020, 10, 2309-2326. [CrossRef]

5. Yadav, K.K. How AI Is Optimizing the Detection and Management of Prostate Cancer. IEEE Pulse 2018, 9, 19. [CrossRef]

6. Russell Stuart, J.; Norvig, P. Artificial Intelligence: A Modern Approach; Prentice Hall: Upper Saddle River, NJ, USA, 2009; ISBN 0-13-207148-7.

7. Goldenberg, S.L.; Nir, G.; Salcudean, S.E. A New Era: Artificial Intelligence and Machine Learning in Prostate Cancer. Nat. Rev. Urol. 2019, 16, 391-403. [CrossRef] [PubMed]

8. Goodfellow, I.; Bengio, Y.; Courville, A.; Bengio, Y. Deep Learning; MIT Press: Cambridge, UK, 2016; Volume 1.

9. Hussain, L.; Ahmed, A.; Saeed, S.; Rathore, S.; Awan, I.A.; Shah, S.A.; Majid, A.; Idris, A.; Awan, A.A. Prostate Cancer Detection Using Machine Learning Techniques by Employing Combination of Features Extracting Strategies. Cancer Biomark. 2018, 21, 393-413. [CrossRef]

10. Song, Y.; Zhang, Y.-D.; Yan, X.; Liu, H.; Zhou, M.; Hu, B.; Yang, G. Computer-Aided Diagnosis of Prostate Cancer Using a Deep Convolutional Neural Network from Multiparametric MRI. J. Magn. Reson. Imaging 2018, 48, 1570-1577. [CrossRef]

11. Litjens, G.; Kooi, T.; Bejnordi, B.E.; Setio, A.A.A.; Ciompi, F.; Ghafoorian, M.; van der Laak, J.A.W.M.; van Ginneken, B.; Sánchez, C.I. A Survey on Deep Learning in Medical Image Analysis. Med. Image Anal. 2017, 42, 60-88. [CrossRef] [PubMed]

12. Shen, D.; Wu, G.; Suk, H.-I. Deep Learning in Medical Image Analysis. Annu. Rev. Biomed. Eng. 2017, 19, 221-248. [CrossRef] [PubMed]

13. Suzuki, K. Overview of Deep Learning in Medical Imaging. Radiol. Phys. Technol. 2017, 10, 257-273. [CrossRef]

14. Van Booven, D.J.; Kuchakulla, M.; Pai, R.; Frech, F.S.; Ramasahayam, R.; Reddy, P.; Parmar, M.; Ramasamy, R.; Arora, H. A Systematic Review of Artificial Intelligence in Prostate Cancer. Res. Rep. Urol. 2021, 13, 31-39. [CrossRef] [PubMed]

15. Klarenbeek, S.E.; Weekenstroo, H.H.A.; Sedelaar, J.P.M.; Fütterer, J.J.; Prokop, M.; Tummers, M. The Effect of Higher Level Computerized Clinical Decision Support Systems on Oncology Care: A Systematic Review. Cancers 2020, 12, 1032. [CrossRef]

16. Mintz, Y.; Brodie, R. Introduction to Artificial Intelligence in Medicine. Minim. Invasive Ther. Allied Technol. 2019, $28,73-81$. [CrossRef]

17. Bishop, C.M. Pattern Recognition and Machine Learning; Springer: Berlin, Germany, 2006; ISBN 1-4939-3843-6.

18. LeCun, Y.; Bengio, Y.; Hinton, G. Deep Learning. Nature 2015, 521, 436-444. [CrossRef] [PubMed]

19. LeCun, Y.; Boser, B.; Denker, J.S.; Henderson, D.; Howard, R.E.; Hubbard, W.; Jackel, L.D. Backpropagation Applied to Handwritten Zip Code Recognition. Neural Comput. 1989, 1, 541-551. [CrossRef]

20. Pantanowitz, L. Digital Images and the Future of Digital Pathology. J. Pathol. Inform. 2010, 1. [CrossRef] [PubMed]

21. Huisman, A.; Looijen, A.; van den Brink, S.M.; van Diest, P.J. Creation of a Fully Digital Pathology Slide Archive by High-Volume Tissue Slide Scanning. Hum. Pathol. 2010, 41, 751-757. [CrossRef]

22. Chen, P.-H.C.; Gadepalli, K.; MacDonald, R.; Liu, Y.; Kadowaki, S.; Nagpal, K.; Kohlberger, T.; Dean, J.; Corrado, G.S.; Hipp, J.D.; et al. An Augmented Reality Microscope with Real-Time Artificial Intelligence Integration for Cancer Diagnosis. Nat. Med. 2019, 25, 1453-1457. [CrossRef]

23. Montironi, R.; Cheng, L.; Lopez-Beltran, A.; Scarpelli, M. Quantitative Image Analysis on Histologic Virtual Slides for Prostate Pathology Diagnosis, Response to Chemopreventive Agents, and Prognosis. Eur. Urol. Focus 2017, 3, 467-469. [CrossRef]

24. Kwak, J.T.; Hewitt, S.M. Multiview Boosting Digital Pathology Analysis of Prostate Cancer. Comput. Methods Programs Biomed. 2017, 142, 91-99. [CrossRef]

25. Arvaniti, E.; Fricker, K.S.; Moret, M.; Rupp, N.; Hermanns, T.; Fankhauser, C.; Wey, N.; Wild, P.J.; Rüschoff, J.H.; Claassen, M. Automated Gleason Grading of Prostate Cancer Tissue Microarrays via Deep Learning. Sci. Rep. 2018, 8, 12054. [CrossRef]

26. Roffman, D.A.; Hart, G.R.; Leapman, M.S.; Yu, J.B.; Guo, F.L.; Ali, I.; Deng, J. Development and Validation of a Multiparameterized Artificial Neural Network for Prostate Cancer Risk Prediction and Stratification. JCO Clin. Cancer Inform. 2018, 2, 1-10. [CrossRef]

27. Lenain, R.; Seneviratne, M.G.; Bozkurt, S.; Blayney, D.W.; Brooks, J.D.; Hernandez-Boussard, T. Machine Learning Approaches for Extracting Stage from Pathology Reports in Prostate Cancer. Stud. Health Technol. Inform. 2019, 264, 1522-1523. [CrossRef] [PubMed]

28. F-Score. Available online: https:// deepai.org/machine-learning-glossary-and-terms/f-score (accessed on 11 September 2020).

29. Ström, P.; Kartasalo, K.; Olsson, H.; Solorzano, L.; Delahunt, B.; Berney, D.M.; Bostwick, D.G.; Evans, A.J.; Grignon, D.J.; Humphrey, P.A.; et al. Artificial Intelligence for Diagnosis and Grading of Prostate Cancer in Biopsies: A Population-Based, Diagnostic Study. Lancet Oncol. 2020, 21, 222-232. [CrossRef]

30. Litjens, G.; Sánchez, C.I.; Timofeeva, N.; Hermsen, M.; Nagtegaal, I.; Kovacs, I.; Hulsbergen-van de Kaa, C.; Bult, P.; van Ginneken, B.; van der Laak, J. Deep Learning as a Tool for Increased Accuracy and Efficiency of Histopathological Diagnosis. Sci. Rep. 2016, 6, 26286. [CrossRef] [PubMed] 
31. Campanella, G.; Hanna, M.G.; Geneslaw, L.; Miraflor, A.; Werneck Krauss Silva, V.; Busam, K.J.; Brogi, E.; Reuter, V.E.; Klimstra, D.S.; Fuchs, T.J. Clinical-Grade Computational Pathology Using Weakly Supervised Deep Learning on Whole Slide Images. Nat. Med. 2019, 25, 1301-1309. [CrossRef] [PubMed]

32. Campanella, G.; Silva, V.W.K.; Fuchs, T.J. Terabyte-Scale Deep Multiple Instance Learning for Classification and Localization in Pathology. arXiv 2018, arXiv:1805.06983.

33. Nir, G.; Karimi, D.; Goldenberg, S.L.; Fazli, L.; Skinnider, B.F.; Tavassoli, P.; Turbin, D.; Villamil, C.F.; Wang, G.; Thompson, D.J.S.; et al. Comparison of Artificial Intelligence Techniques to Evaluate Performance of a Classifier for Automatic Grading of Prostate Cancer From Digitized Histopathologic Images. JAMA Netw. Open 2019, 2, e190442. [CrossRef] [PubMed]

34. Nir, G.; Hor, S.; Karimi, D.; Fazli, L.; Skinnider, B.F.; Tavassoli, P.; Turbin, D.; Villamil, C.F.; Wang, G.; Wilson, R.S.; et al. Automatic Grading of Prostate Cancer in Digitized Histopathology Images: Learning from Multiple Experts. Med. Image Anal. 2018, 50, 167-180. [CrossRef]

35. Allsbrook, W.C.; Mangold, K.A.; Johnson, M.H.; Lane, R.B.; Lane, C.G.; Amin, M.B.; Bostwick, D.G.; Humphrey, P.A.; Jones, E.C.; Reuter, V.E.; et al. Interobserver Reproducibility of Gleason Grading of Prostatic Carcinoma: Urologic Pathologists. Hum. Pathol. 2001, 32, 74-80. [CrossRef]

36. Nagpal, K.; Foote, D.; Liu, Y.; Chen, P.-H.C.; Wulczyn, E.; Tan, F.; Olson, N.; Smith, J.L.; Mohtashamian, A.; Wren, J.H.; et al. Development and Validation of a Deep Learning Algorithm for Improving Gleason Scoring of Prostate Cancer. NPJ Digit. Med. 2019, 2, 48. [CrossRef]

37. Lucas, M.; Jansen, I.; Savci-Heijink, C.D.; Meijer, S.L.; de Boer, O.J.; van Leeuwen, T.G.; de Bruin, D.M.; Marquering, H.A. Deep Learning for Automatic Gleason Pattern Classification for Grade Group Determination of Prostate Biopsies. Virchows Arch. 2019, 475, 77-83. [CrossRef]

38. Raciti, P.; Sue, J.; Ceballos, R.; Godrich, R.; Kunz, J.D.; Kapur, S.; Reuter, V.; Grady, L.; Kanan, C.; Klimstra, D.S.; et al. Novel Artificial Intelligence System Increases the Detection of Prostate Cancer in Whole Slide Images of Core Needle Biopsies. Mod. Pathol. 2020, 33, 2058-2066. [CrossRef] [PubMed]

39. Bulten, W.; Pinckaers, H.; van Boven, H.; Vink, R.; de Bel, T.; van Ginneken, B.; van der Laak, J.; Hulsbergen-van de Kaa, C.; Litjens, G. Automated Deep-Learning System for Gleason Grading of Prostate Cancer Using Biopsies: A Diagnostic Study. Lancet Oncol. 2020, 21, 233-241. [CrossRef]

40. Egevad, L.; Swanberg, D.; Delahunt, B.; Ström, P.; Kartasalo, K.; Olsson, H.; Berney, D.M.; Bostwick, D.G.; Evans, A.J.; Humphrey, P.A.; et al. Identification of Areas of Grading Difficulties in Prostate Cancer and Comparison with Artificial Intelligence Assisted Grading. Virchows Arch. 2020. [CrossRef] [PubMed]

41. Bulten, W.; Balkenhol, M.; Belinga, J.-J.A.; Brilhante, A.; Çakır, A.; Egevad, L.; Eklund, M.; Farré, X.; Geronatsiou, K.; Molinié, V.; et al. Artificial Intelligence Assistance Significantly Improves Gleason Grading of Prostate Biopsies by Pathologists. Mod. Pathol. 2020. [CrossRef]

42. Schnall, M.D.; Imai, Y.; Tomaszewski, J.; Pollack, H.M.; Lenkinski, R.E.; Kressel, H.Y. Prostate Cancer: Local Staging with Endorectal Surface Coil MR Imaging. Radiology 1991, 178, 797-802. [CrossRef]

43. Ward, A.D.; Crukley, C.; McKenzie, C.A.; Montreuil, J.; Gibson, E.; Romagnoli, C.; Gomez, J.A.; Moussa, M.; Chin, J.; Bauman, G.; et al. Prostate: Registration of Digital Histopathologic Images to in Vivo MR Images Acquired by Using Endorectal Receive Coil. Radiology 2012, 263, 856-864. [CrossRef]

44. Litjens, G.; Toth, R.; van de Ven, W.; Hoeks, C.; Kerkstra, S.; van Ginneken, B.; Vincent, G.; Guillard, G.; Birbeck, N.; Zhang, J.; et al. Evaluation of Prostate Segmentation Algorithms for MRI: The PROMISE12 Challenge. Med. Image Anal. 2014, 18, 359-373. [CrossRef]

45. Gaur, S.; Lay, N.; Harmon, S.A.; Doddakashi, S.; Mehralivand, S.; Argun, B.; Barrett, T.; Bednarova, S.; Girometti, R.; Karaarslan, E.; et al. Can Computer-Aided Diagnosis Assist in the Identification of Prostate Cancer on Prostate MRI? A Multi-Center, Multi-Reader Investigation. Oncotarget 2018, 9, 33804-33817. [CrossRef] [PubMed]

46. Abdollahi, H.; Mofid, B.; Shiri, I.; Razzaghdoust, A.; Saadipoor, A.; Mahdavi, A.; Galandooz, H.M.; Mahdavi, S.R. Machine Learning-Based Radiomic Models to Predict Intensity-Modulated Radiation Therapy Response, Gleason Score and Stage in Prostate Cancer. Radiol. Med. 2019, 124, 555-567. [CrossRef] [PubMed]

47. Dulhanty, C.; Wang, L.; Cheng, M.; Gunraj, H.; Khalvati, F.; Haider, M.A.; Wong, A. Radiomics Driven Diffusion Weighted Imaging Sensing Strategies for Zone-Level Prostate Cancer Sensing. Sensors 2020, 20, 1539. [CrossRef] [PubMed]

48. Aldoj, N.; Lukas, S.; Dewey, M.; Penzkofer, T. Semi-Automatic Classification of Prostate Cancer on Multi-Parametric MR Imaging Using a Multi-Channel 3D Convolutional Neural Network. Eur. Radiol. 2020, 30, 1243-1253. [CrossRef]

49. Kasel-Seibert, M.; Lehmann, T.; Aschenbach, R.; Guettler, F.V.; Abubrig, M.; Grimm, M.-O.; Teichgraeber, U.; Franiel, T. Assessment of PI-RADS v2 for the Detection of Prostate Cancer. Eur. J. Radiol. 2016, 85, 726-731. [CrossRef] [PubMed]

50. Armato, S.G.; Huisman, H.; Drukker, K.; Hadjiiski, L.; Kirby, J.S.; Petrick, N.; Redmond, G.; Giger, M.L.; Cha, K.; Mamonov, A.; et al. PROSTATEx Challenges for Computerized Classification of Prostate Lesions from Multiparametric Magnetic Resonance Images. J. Med Imaging 2018, 5, 044501. [CrossRef]

51. De Vente, C.; Vos, P.; Hosseinzadeh, M.; Pluim, J.; Veta, M. Deep Learning Regression for Prostate Cancer Detection and Grading in Bi-Parametric MRI. IEEE Trans. Biomed. Eng. 2020. [CrossRef] 
52. Zong, W.; Lee, J.K.; Liu, C.; Carver, E.N.; Feldman, A.M.; Janic, B.; Elshaikh, M.A.; Pantelic, M.V.; Hearshen, D.; Chetty, I.J.; et al. A Deep Dive into Understanding Tumor Foci Classification Using Multiparametric MRI Based on Convolutional Neural Network. Med. Phys. 2020. [CrossRef]

53. Sunoqrot, M.R.S.; Nketiah, G.A.; Selnæs, K.M.; Bathen, T.F.; Elschot, M. Automated Reference Tissue Normalization of T2Weighted MR Images of the Prostate Using Object Recognition. MAGMA 2020. [CrossRef]

54. Sanford, T.; Harmon, S.A.; Turkbey, E.B.; Kesani, D.; Tuncer, S.; Madariaga, M.; Yang, C.; Sackett, J.; Mehralivand, S.; Yan, P.; et al Deep-Learning-Based Artificial Intelligence for PI-RADS Classification to Assist Multiparametric Prostate MRI Interpretation: A Development Study. J. Magn. Reson. Imaging 2020. [CrossRef]

55. Chatterjee, A.; Harmath, C.; Oto, A. New Prostate MRI Techniques and Sequences. Abdom. Radiol. 2020. [CrossRef]

56. Harmon, S.A.; Tuncer, S.; Sanford, T.; Choyke, P.L.; Türkbey, B. Artificial Intelligence at the Intersection of Pathology and Radiology in Prostate Cancer. Diagn. Interv. Radiol. 2019, 25, 183-188. [CrossRef] [PubMed]

57. Greer, M.D.; Lay, N.; Shih, J.H.; Barrett, T.; Bittencourt, L.K.; Borofsky, S.; Kabakus, I.; Law, Y.M.; Marko, J.; Shebel, H.; et al. Computer-Aided Diagnosis Prior to Conventional Interpretation of Prostate MpMRI: An International Multi-Reader Study. Eur. Radiol. 2018, 28, 4407-4417. [CrossRef] [PubMed]

58. Zeng, Q.; Samei, G.; Karimi, D.; Kesch, C.; Mahdavi, S.S.; Abolmaesumi, P.; Salcudean, S.E. Prostate Segmentation in Transrectal Ultrasound Using Magnetic Resonance Imaging Priors. Int. J. Comput. Assist. Radiol. Surg. 2018, 13, 749-757. [CrossRef] [PubMed]

59. Karimi, D.; Zeng, Q.; Mathur, P.; Avinash, A.; Mahdavi, S.; Spadinger, I.; Abolmaesumi, P.; Salcudean, S.E. Accurate and Robust Deep Learning-Based Segmentation of the Prostate Clinical Target Volume in Ultrasound Images. Med. Image Anal. 2019, 57, 186-196. [CrossRef]

60. Feng, Y.; Yang, F.; Zhou, X.; Guo, Y.; Tang, F.; Ren, F.; Guo, J.; Ji, S. A Deep Learning Approach for Targeted Contrast-Enhanced Ultrasound Based Prostate Cancer Detection. IEEE/ACM Trans. Comput. Biol. Bioinform. 2019, 16, 1794-1801. [CrossRef]

61. Wildeboer, R.R.; Mannaerts, C.K.; van Sloun, R.J.G.; Budäus, L.; Tilki, D.; Wijkstra, H.; Salomon, G.; Mischi, M. Automated Multiparametric Localization of Prostate Cancer Based on B-Mode, Shear-Wave Elastography, and Contrast-Enhanced Ultrasound Radiomics. Eur. Radiol. 2020, 30, 806-815. [CrossRef]

62. Anas, E.M.A.; Nouranian, S.; Mahdavi, S.S.; Spadinger, I.; Morris, W.J.; Salcudean, S.E.; Mousavi, P.; Abolmaesumi, P. Clinical Target-Volume Delineation in Prostate Brachytherapy Using Residual Neural Networks. In Proceedings of the International Conference on Medical Image Computing and Computer-Assisted Intervention; Springer: Berlin, Germany, 2017; pp. 365-373.

63. Anas, E.M.A.; Mousavi, P.; Abolmaesumi, P. A Deep Learning Approach for Real Time Prostate Segmentation in Freehand Ultrasound Guided Biopsy. Med. Image Anal. 2018, 48, 107-116. [CrossRef]

64. Hu, Y.; Modat, M.; Gibson, E.; Li, W.; Ghavami, N.; Bonmati, E.; Wang, G.; Bandula, S.; Moore, C.M.; Emberton, M.; et al. Weakly-Supervised Convolutional Neural Networks for Multimodal Image Registration. Med. Image Anal. 2018, 49, 1-13. [CrossRef]

65. Ushinsky, A.; Bardis, M.; Glavis-Bloom, J.; Uchio, E.; Chantaduly, C.; Nguyentat, M.; Chow, D.; Chang, P.; Houshyar, R. A 3D/2D Hybrid U-Net CNN Approach to Prostate Organ Segmentation of MpMRI. AJR Am. J. Roentgenol. 2020. [CrossRef]

66. Liu, B.; Cheng, J.; Guo, D.J.; He, X.J.; Luo, Y.D.; Zeng, Y.; Li, C.M. Prediction of Prostate Cancer Aggressiveness with a Combination of Radiomics and Machine Learning-Based Analysis of Dynamic Contrast-Enhanced MRI. Clin. Radiol. 2019, 74, 896.e1-896.e8. [CrossRef]

67. Ishioka, J.; Matsuoka, Y.; Uehara, S.; Yasuda, Y.; Kijima, T.; Yoshida, S.; Yokoyama, M.; Saito, K.; Kihara, K.; Numao, N.; et al. Computer-Aided Diagnosis of Prostate Cancer on Magnetic Resonance Imaging Using a Convolutional Neural Network Algorithm. BJU Int. 2018, 122, 411-417. [CrossRef] [PubMed]

68. Checcucci, E.; Autorino, R.; Cacciamani, G.E.; Amparore, D.; De Cillis, S.; Piana, A.; Piazzolla, P.; Vezzetti, E.; Fiori, C.; Veneziano, D.; et al. Artificial Intelligence and Neural Networks in Urology: Current Clinical Applications. Minerva Urol. Nefrol. 2020, 72, 49-57. [CrossRef] [PubMed]

69. Bertoli, G.; Cava, C.; Castiglioni, I. MicroRNAs as Biomarkers for Diagnosis, Prognosis and Theranostics in Prostate Cancer. Int. J. Mol. Sci. 2016, 17, 421. [CrossRef] [PubMed]

70. MacInnis, R.J.; Schmidt, D.F.; Makalic, E.; Severi, G.; FitzGerald, L.M.; Reumann, M.; Kapuscinski, M.K.; Kowalczyk, A.; Zhou, Z.; Goudey, B.; et al. Use of a Novel Nonparametric Version of DEPTH to Identify Genomic Regions Associated with Prostate Cancer Risk. Cancer Epidemiol. Biomark. Prev. 2016, 25, 1619-1624. [CrossRef]

71. Karnes, R.J.; Bergstralh, E.J.; Davicioni, E.; Ghadessi, M.; Buerki, C.; Mitra, A.P.; Crisan, A.; Erho, N.; Vergara, I.A.; Lam, L.L.; et al Validation of a Genomic Classifier That Predicts Metastasis Following Radical Prostatectomy in an at Risk Patient Population. J. Urol. 2013, 190, 2047-2053. [CrossRef]

72. Lee, S.; Kerns, S.; Ostrer, H.; Rosenstein, B.; Deasy, J.O.; Oh, J.H. Machine Learning on a Genome-Wide Association Study to Predict Late Genitourinary Toxicity After Prostate Radiation Therapy. Int. J. Radiat. Oncol. Biol. Phys. 2018, 101, 128-135. [CrossRef]

73. Hou, Q.; Bing, Z.-T.; Hu, C.; Li, M.-Y.; Yang, K.-H.; Mo, Z.; Xie, X.-W.; Liao, J.-L.; Lu, Y.; Horie, S.; et al. RankProd Combined with Genetic Algorithm Optimized Artificial Neural Network Establishes a Diagnostic and Prognostic Prediction Model That Revealed C1QTNF3 as a Biomarker for Prostate Cancer. EBioMedicine 2018, 32, 234-244. [CrossRef]

74. Liu, B.; Liu, Y.; Pan, X.; Li, M.; Yang, S.; Li, S.C. DNA Methylation Markers for Pan-Cancer Prediction by Deep Learning. Genes 2019, 10, 778. [CrossRef] 
75. Hamzeh, O.; Alkhateeb, A.; Zheng, J.; Kandalam, S.; Rueda, L. Prediction of Tumor Location in Prostate Cancer Tissue Using a Machine Learning System on Gene Expression Data. BMC Bioinform. 2020, 21, 78. [CrossRef]

76. de la Calle, C.M.; Nguyen, H.G.; Hosseini-Asl, E.; So, C.; Socher, R.; Xiong, C.; Xue, L.; Carroll, P.; Cooperberg, M.R. Artificial Intelligence for Streamlined Immunofluorescence-Based Biomarker Discovery in Prostate Cancer. JCO 2020, 38, 279. [CrossRef]

77. Shiradkar, R.; Podder, T.K.; Algohary, A.; Viswanath, S.; Ellis, R.J.; Madabhushi, A. Radiomics Based Targeted Radiotherapy Planning (Rad-TRaP): A Computational Framework for Prostate Cancer Treatment Planning with MRI. Radiat. Oncol. 2016, 11, 148. [CrossRef]

78. Lei, Y.; Tian, S.; He, X.; Wang, T.; Wang, B.; Patel, P.; Jani, A.B.; Mao, H.; Curran, W.J.; Liu, T.; et al. Ultrasound Prostate Segmentation Based on Multidirectional Deeply Supervised V-Net. Med. Phys. 2019, 46, 3194-3206. [CrossRef]

79. Nouranian, S.; Ramezani, M.; Spadinger, I.; Morris, W.J.; Salcudean, S.E.; Abolmaesumi, P. Learning-Based Multi-Label Segmentation of Transrectal Ultrasound Images for Prostate Brachytherapy. IEEE Trans. Med. Imaging 2016, 35, 921-932. [CrossRef]

80. Nicolae, A.; Morton, G.; Chung, H.; Loblaw, A.; Jain, S.; Mitchell, D.; Lu, L.; Helou, J.; Al-Hanaqta, M.; Heath, E.; et al. Evaluation of a Machine-Learning Algorithm for Treatment Planning in Prostate Low-Dose-Rate Brachytherapy. Int. J. Radiat. Oncol. Biol. Phys. 2017, 97, 822-829. [CrossRef]

81. Sanders, J.W.; Frank, S.J.; Kudchadker, R.J.; Bruno, T.L.; Ma, J. Development and Clinical Implementation of SeedNet: A SlidingWindow Convolutional Neural Network for Radioactive Seed Identification in MRI-Assisted Radiosurgery (MARS). Magn. Reson. Med. 2019, 81, 3888-3900. [CrossRef] [PubMed]

82. Sarikaya, D.; Corso, J.J.; Guru, K.A. Detection and Localization of Robotic Tools in Robot-Assisted Surgery Videos Using Deep Neural Networks for Region Proposal and Detection. IEEE Trans. Med. Imaging 2017, 36, 1542-1549. [CrossRef] [PubMed]

83. Hung, A.J.; Chen, J.; Gill, I.S. Automated Performance Metrics and Machine Learning Algorithms to Measure Surgeon Performance and Anticipate Clinical Outcomes in Robotic Surgery. JAMA Surg. 2018, 153, 770-771. [CrossRef]

84. Dong, X.; Lei, Y.; Tian, S.; Wang, T.; Patel, P.; Curran, W.J.; Jani, A.B.; Liu, T.; Yang, X. Synthetic MRI-Aided Multi-Organ Segmentation on Male Pelvic CT Using Cycle Consistent Deep Attention Network. Radiother. Oncol. 2019, 141, 192-199. [CrossRef] [PubMed]

85. Savenije, M.H.F.; Maspero, M.; Sikkes, G.G.; van der Voort van Zyp, J.R.N.; Kotte, A.N.; Bol, G.H.; van den Berg, C.A. Clinical Implementation of MRI-Based Organs-at-Risk Auto-Segmentation with Convolutional Networks for Prostate Radiotherapy. Radiat. Oncol. 2020, 15, 104. [CrossRef]

86. Shafai-Erfani, G.; Wang, T.; Lei, Y.; Tian, S.; Patel, P.; Jani, A.B.; Curran, W.J.; Liu, T.; Yang, X. Dose Evaluation of MRI-Based Synthetic CT Generated Using a Machine Learning Method for Prostate Cancer Radiotherapy. Med. Dosim. 2019, 44, e64-e70. [CrossRef] [PubMed]

87. Nosrati, R.; Soliman, A.; Safigholi, H.; Hashemi, M.; Wronski, M.; Morton, G.; Pejović-Milić, A.; Stanisz, G.; Song, W.Y. MRI-Based Automated Detection of Implanted Low Dose Rate (LDR) Brachytherapy Seeds Using Quantitative Susceptibility Mapping (QSM) and Unsupervised Machine Learning (ML). Radiother. Oncol. 2018, 129, 540-547. [CrossRef]

88. Mao, X.; Pineau, J.; Keyes, R.; Enger, S.A. RapidBrachyDL: Rapid Radiation Dose Calculations in Brachytherapy Via Deep Learning. Int. J. Radiat. Oncol. Biol. Phys. 2020, 108, 802-812. [CrossRef]

89. Hrinivich, W.T.; Lee, J. Artificial Intelligence-Based Radiotherapy Machine Parameter Optimization Using Reinforcement Learning. Med. Phys. 2020. [CrossRef]

90. Xing, Y.; Nguyen, D.; Lu, W.; Yang, M.; Jiang, S. Technical Note: A Feasibility Study on Deep Learning-Based Radiotherapy Dose Calculation. Med. Phys. 2020, 47, 753-758. [CrossRef] [PubMed]

91. Isaksson, L.J.; Pepa, M.; Zaffaroni, M.; Marvaso, G.; Alterio, D.; Volpe, S.; Corrao, G.; Augugliaro, M.; Starzyńska, A.; Leonardi, M.C.; et al. Machine Learning-Based Models for Prediction of Toxicity Outcomes in Radiotherapy. Front. Oncol. 2020, 10, 790. [CrossRef] [PubMed]

92. Checcucci, E.; De Cillis, S.; Granato, S.; Chang, P.; Afyouni, A.S.; Okhunov, Z. Uro-technology and SoMe Working Group of the Young Academic Urologists Working Party of the European Association of Urology Applications of Neural Networks in Urology: A Systematic Review. Curr. Opin. Urol. 2020, 30, 788-807. [CrossRef]

93. Kassahun, Y.; Yu, B.; Tibebu, A.T.; Stoyanov, D.; Giannarou, S.; Metzen, J.H.; Vander Poorten, E. Surgical Robotics beyond Enhanced Dexterity Instrumentation: A Survey of Machine Learning Techniques and Their Role in Intelligent and Autonomous Surgical Actions. Int. J. Comput. Assist. Radiol. Surg. 2016, 11, 553-568. [CrossRef] [PubMed]

94. Ranasinghe, W.; de Silva, D.; Bandaragoda, T.; Adikari, A.; Alahakoon, D.; Persad, R.; Lawrentschuk, N.; Bolton, D. RoboticAssisted vs. Open Radical Prostatectomy: A Machine Learning Framework for Intelligent Analysis of Patient-Reported Outcomes from Online Cancer Support Groups. Urol. Oncol. 2018, 36, 529.e1-529.e9. [CrossRef]

95. Wong, N.C.; Lam, C.; Patterson, L.; Shayegan, B. Use of Machine Learning to Predict Early Biochemical Recurrence after Robot-Assisted Prostatectomy. BJU Int. 2019, 123, 51-57. [CrossRef]

96. Colling, R.; Pitman, H.; Oien, K.; Rajpoot, N.; Macklin, P.; CM-Path AI in Histopathology Working Group; Snead, D.; Sackville, T.; Verrill, C. Artificial Intelligence in Digital Pathology: A Roadmap to Routine Use in Clinical Practice. J. Pathol. 2019, 249, 143-150. [CrossRef]

97. Smith, J.A.; Abhari, R.E.; Hussain, Z.; Heneghan, C.; Collins, G.S.; Carr, A.J. Industry Ties and Evidence in Public Comments on the FDA Framework for Modifications to Artificial Intelligence/Machine Learning-Based Medical Devices: A Cross Sectional Study. BMJ Open 2020, 10, e039969. [CrossRef] [PubMed] 
98. Acs, B.; Rantalainen, M.; Hartman, J. Artificial Intelligence as the next Step towards Precision Pathology. J. Intern. Med. 2020, 288, 62-81. [CrossRef] [PubMed]

99. Wildeboer, R.R.; van Sloun, R.J.G.; Wijkstra, H.; Mischi, M. Artificial Intelligence in Multiparametric Prostate Cancer Imaging with Focus on Deep-Learning Methods. Comput. Methods Programs Biomed. 2020, 189, 105316. [CrossRef]

100. Kather, J.N.; Weidner, A.; Attenberger, U.; Bukschat, Y.; Weis, C.-A.; Weis, M.; Schad, L.R.; Zöllner, F.G. Color-Coded Visualization of Magnetic Resonance Imaging Multiparametric Maps. Sci. Rep. 2017, 7, 41107. [CrossRef] [PubMed]

101. Wegelin, O.; van Melick, H.H.E.; Hooft, L.; Bosch, J.L.H.R.; Reitsma, H.B.; Barentsz, J.O.; Somford, D.M. Comparing Three Different Techniques for Magnetic Resonance Imaging-Targeted Prostate Biopsies: A Systematic Review of In-Bore versus Magnetic Resonance Imaging-Transrectal Ultrasound Fusion versus Cognitive Registration. Is There a Preferred Technique? Eur. Urol. 2017, 71, 517-531. [CrossRef] [PubMed] 